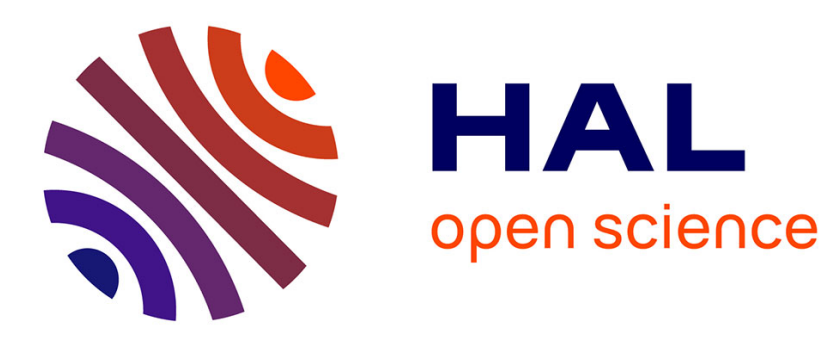

\title{
Mild and Metal-Free Diastereoselective Synthesis of N-tert-Butanesulfinylamines by Using Tetrakis(dimethylamino)ethylene
}

Cédric Spitz, Anna Lin, Thierry Terme, Patrice Vanelle

\section{To cite this version:}

Cédric Spitz, Anna Lin, Thierry Terme, Patrice Vanelle. Mild and Metal-Free Diastereoselective Synthesis of N-tert-Butanesulfinylamines by Using Tetrakis(dimethylamino)ethylene. Synthesis: Journal of Synthetic Organic Chemistry, 2014, 10.1055/s-0034-1378636 . hal-01425582

\section{HAL Id: hal-01425582 \\ https://hal-amu.archives-ouvertes.fr/hal-01425582}

Submitted on 3 Jan 2017

HAL is a multi-disciplinary open access archive for the deposit and dissemination of scientific research documents, whether they are published or not. The documents may come from teaching and research institutions in France or abroad, or from public or private research centers.
L'archive ouverte pluridisciplinaire HAL, est destinée au dépôt et à la diffusion de documents scientifiques de niveau recherche, publiés ou non, émanant des établissements d'enseignement et de recherche français ou étrangers, des laboratoires publics ou privés. 


\title{
Mild and Metal-Free Diastereoselective Synthesis of $\boldsymbol{N}$-tert-Butanesulfinylamines by Using Tetrakis(dimethylamino)ethylene
}

\author{
Cédric Spitz, Anna Lin, Thierry Terme, Patrice Vanelle* \\ Aix-Marseille Université, CNRS, ICR UMR 7273, Equipe Pharmaco-Chimie Radicalaire, Faculté de Pharmacie, 27 Boulevard Jean Moulin - \\ CS 30064 - 13385 Marseille Cedex 05, France \\ Fax+33(4)91794677; E-mail: patrice.vanelle@univ-amu.fr \\ Received: 19.05.2014; Accepted after revision: 18.07.2014
}

\begin{abstract}
A mild and metal-free diastereoselective synthesis of $N$ tert-butanesulfinylamines was developed by using a strategy based on tetrakis(dimethylamino)ethylene. Good yields and diastereoselectivities were achieved by addition of $o$-nitrobenzyl chloride or 4-[4-(chloromethyl)phenyl]-1,2-dimethyl-5-nitro- $1 H$-imidazole to readily available $N$-tert-butanesulfinimines.
\end{abstract}

Key words: sulfinimines, diastereoselectivity, amines, metal-free, chiral auxiliary

The asymmetric synthesis of amines has received considerable attention in recent years because nitrogen-containing molecules are ubiquitous components of biologically active compounds. ${ }^{1}$ Adding a nucleophile to enantiomerically pure $N$-tert-butanesulfinimines is one of the most commonly used methods for the asymmetric synthesis of amines. ${ }^{2}$ In particular, adding organometallic reagents to $N$-sulfinimines usually leads to the synthesis of amine products in good yields and diastereoselectivities. Furthermore, the tert-butanesulfinyl chiral auxiliary can be removed easily under acidic conditions, giving the corresponding chiral primary amines. However, the disadvantage of using organometallic reagents such as Grignard or organolithium reagents is their poor functional group tolerance. Recently, Ellman and co-workers reported the $\mathrm{MgCl}_{2}$-enhanced addition of benzyl zinc reagents to $\mathrm{N}$ tert-butanesulfinimines, obtaining good yields, diastereoselectivities and better functional group tolerance. ${ }^{3}$ However, to our knowledge, the addition of organometallic reagents bearing a nitro group to $N$-tert-butanesulfinimines has never been described, probably due to the difficulty of generating organometallic nitro compounds. ${ }^{4}$

Tetrakis(dimethylamino)ethylene (TDAE) is an organic reducing agent that reacts with halogenated derivatives to generate a carbanion under mild conditions. ${ }^{5}$ Since 2003, our research has focused on developing original synthetic methods using TDAE methodology in medicinal chemistry. ${ }^{6}$ Recently, we reported the application of our TDAE methodology to the diastereoselective addition of $p$-nitrobenzyl chloride to a variety of enantiopure $(R)-N$-tert-butanesulfinimines, producing chiral amines in good yields and diastereoselectivities. ${ }^{7}$

SYNTHESIS 2014, 46, 3229-3232

Advanced online publication: 27.08.2014

DOI: 10.1055/s-0034-1378636; Art ID: ss-2014-z0307-op

(C) Georg Thieme Verlag Stuttgart · New York
As part of our research program on new bioactive compounds, ${ }^{8}$ we report herein the diastereoselective synthesis of chiral amines through the addition of either $o$-nitrobenzyl chloride (1) or 4-[4-(chloromethyl)phenyl]-1,2-dimethyl-5-nitro- $1 \mathrm{H}$-imidazole (4) to enantiopure $(R)-\mathrm{N}$ tert-butanesulfinimines using TDAE.

First, to compare the influence of an ortho substituent with that of a para substituent on the benzyl chloride system, the commercially available 1 was used in the reaction with sulfinimines $\mathbf{2 a}-\mathbf{g}$ in the presence of TDAE at $-20{ }^{\circ} \mathrm{C}$ for one hour, followed by 20 hours at room temperature. Under these conditions, a variety of $N$-tert-butanesulfinylamines were obtained in good yields (52-66\%) and with good diastereoselectivities (from 83:17 to 89:11). The results are summarised in Table 1. Sulfinimines with para-, meta-, and ortho-substitution were all well tolerated and, encouragingly, the substituents did not affect diastereoselectivity (entries 1-6). Interestingly, the use of heteroaro-

Table 1 Diastereoselective Addition of 1 to (Hetero)Aromatic Sulfinimines 2a-g by Using the TDAE Strategy ${ }^{\mathrm{a}}$

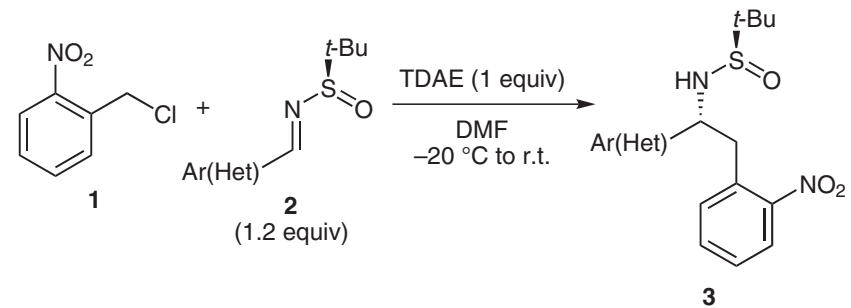

\begin{tabular}{|c|c|c|c|c|}
\hline Entry & Imine 2 & $\operatorname{Ar}(\mathrm{Het})$ & Yield $(\%)^{b}$ of $\mathbf{3}$ & $\mathrm{dr}^{\mathrm{c}}$ \\
\hline 1 & $2 a$ & $4-\mathrm{O}_{2} \mathrm{NC}_{6} \mathrm{H}_{4}$ & 66 & $89: 11$ \\
\hline 2 & $2 \mathbf{b}$ & $3-\mathrm{O}_{2} \mathrm{NC}_{6} \mathrm{H}_{4}$ & 60 & $88: 12$ \\
\hline 3 & $2 c$ & $2-\mathrm{O}_{2} \mathrm{NC}_{6} \mathrm{H}_{4}$ & 59 & $87: 13$ \\
\hline 4 & 2d & $4-\mathrm{BrC}_{6} \mathrm{H}_{4}$ & 52 & $89: 11$ \\
\hline 5 & $2 e$ & 4- $\mathrm{NCC}_{6} \mathrm{H}_{4}$ & 62 & $89: 11$ \\
\hline 6 & $2 f$ & $3-\mathrm{NCC}_{6} \mathrm{H}_{4}$ & 65 & $83: 17$ \\
\hline 7 & $2 \mathrm{~g}$ & 2-pyridyl & 59 & $87: 13$ \\
\hline
\end{tabular}

${ }^{a}$ Reaction conditions: chloride 1 ( 1 equiv), imine 2 ( 1.2 equiv),

TDAE ( 1 equiv), anhydrous DMF, $-20^{\circ} \mathrm{C}, 1 \mathrm{~h}$, then r.t., $20 \mathrm{~h}$.

${ }^{\mathrm{b}}$ Combined yield of diastereoisomers after purification by chromatography.

${ }^{\mathrm{c}}$ Determined after separation of the two diastereoisomers by chromatography. 
matic imine $2 \mathrm{~g}$ allowed the formation of the corresponding amine $\mathbf{3 g}$ in good yield and diastereoselectivity (entry 7).

To extend the scope of the reaction to other benzyl chloride derivatives containing a nitro group and because the 5-nitroimidazole moiety is well known to exhibit a wide spectrum of anti-infectious activity, ${ }^{9}$ we then performed the reaction between 4 and various enantiopure (hetero)aromatic $N$-sulfinimines $\mathbf{2 a}, \mathbf{2 d - e}$, and $\mathbf{2 g - k}$. In the presence of TDAE, the corresponding amines were obtained in moderate to good yields (41-78\%) and good diastereoselectivities (from $82: 18$ to $89: 11$ ), as shown in Table 2. Reactions with electron-poor aromatic imines produced good yields and good diastereoselectivities (entries 1-3 and 5-6). The diastereoselectivity obtained with the unsubstituted imine $\mathbf{2} \mathbf{j}$ was still good but, as expected, the yield was slightly diminished (entry 7). The electronrich imine $\mathbf{2 k}$, with a methoxy substituent, also gave good diastereoselectivity, and $44 \%$ yield was obtained by using imine 2k ( 1 equiv), chloride 4 ( 3 equiv), and TDAE ( 3 equiv; entry 8). Again, it is interesting to note that the use of heteroaromatic imine $\mathbf{2 g}$ allowed the formation of the corresponding amine $\mathbf{5 g}$ in good yield and diastereoselectivity (entry 4). It should be noted that a bis-heteroaromatic compound such as the latter would be very difficult to synthesise by using organometallic chemistry.

The absolute configuration of the major diastereoisomer was assigned as $R, R$ by analogy with our previous communication. $^{6}$

In summary, a mild and metal-free diastereoselective synthesis of $N$-tert-butanesulfinylamines was developed by using a TDAE strategy. The tolerance of nitro groups proved that this method is a good alternative to the use of organometallic reagents to prepare enantiopure amines containing nitro substituents.

All chemicals and reagents were purchased from commercial suppliers. Melting points were determined with a Büchi melting point B-540 apparatus and are uncorrected. Both ${ }^{1} \mathrm{H}$ and ${ }^{13} \mathrm{C}$ NMR spectra were determined with Bruker AC 200, Bruker 300 Avance III nanobay and Bruker 400 Avance III nanobay spectrometers. The ${ }^{1} \mathrm{H}$ and the ${ }^{13} \mathrm{C}$ chemical shifts are reported from $\mathrm{CDCl}_{3}$ peaks: ${ }^{1} \mathrm{H}(\delta=7.26$ $\mathrm{ppm})$ and ${ }^{13} \mathrm{C}(\delta=77.16 \mathrm{ppm})$. Multiplicities are represented by the following notations: singlet $(\mathrm{s})$, doublet $(\mathrm{d})$, triplet $(\mathrm{t})$, quartet $(\mathrm{q})$, and complex multiplet or overlapping multiplets (m). HRMS analyses were recorded with a SYNAPT G2HDMS (Waters) apparatus at the spectropole of the Aix-Marseille University. The following adsorbents were used for column chromatography: silica gel 60 (Merck, particle size $0.063-0.200 \mathrm{~mm}, 70-230$ mesh ASTM). TLC was performed on $5 \times 10 \mathrm{~cm}$ aluminium plates coated with silica gel 60 F254 (Merck) in an appropriate solvent.

\section{General Procedure}

To a stirred solution of $N$-sulfinimine $2(0.24 \mathrm{mmol})$ in anhydrous DMF $(1 \mathrm{~mL})$ at $-20^{\circ} \mathrm{C}$ was added TDAE $(0.2 \mathrm{mmol})$ followed by dropwise addition of a solution of $\mathbf{1}$ or $\mathbf{4}$ in anhydrous DMF $(1 \mathrm{~mL})$. The solution was vigorously stirred at $-20^{\circ} \mathrm{C}$ for $1 \mathrm{~h}$ and then maintained at r.t. for $20 \mathrm{~h} . \mathrm{H}_{2} \mathrm{O}(5 \mathrm{~mL})$ was added and the aqueous solution was extracted with $\mathrm{CH}_{2} \mathrm{Cl}_{2}(3 \times 15 \mathrm{~mL})$. The combined organic layer was washed with $\mathrm{H}_{2} \mathrm{O}(20 \mathrm{~mL})$ and dried over $\mathrm{MgSO}_{4}$. Evaporation of the solvent furnished the crude product, which was puri-
Table 2 Diastereoselective Addition of 4 to (Hetero)Aromatic Sulfinimines 2 by Using the TDAE Strategy ${ }^{\mathrm{a}}$

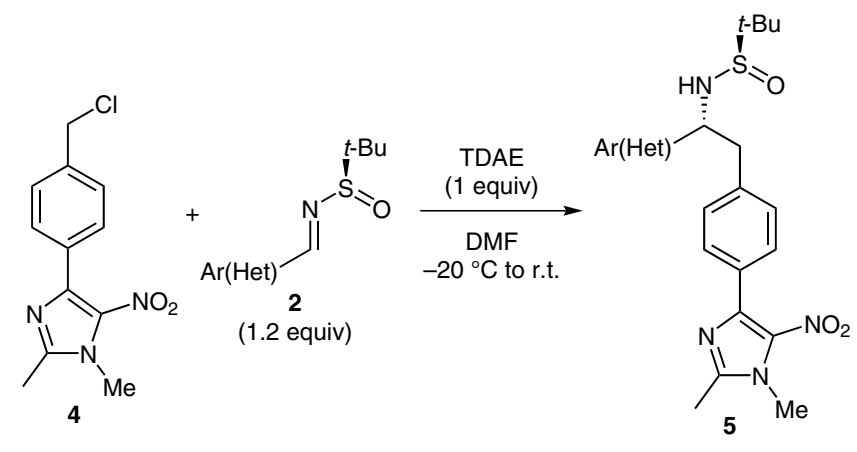

\begin{tabular}{lllll}
\hline Entry & Imine 2 & $\mathrm{Ar}(\mathrm{Het})$ & Yield (\%) of $\mathbf{5}$ & $\mathrm{dr}^{\mathbf{c}}$ \\
\hline 1 & $\mathbf{2 a}$ & $4-\mathrm{O}_{2} \mathrm{NC}_{6} \mathrm{H}_{4}$ & 57 & $82: 18$ \\
2 & $\mathbf{2 d}$ & $4-\mathrm{BrC}_{6} \mathrm{H}_{4}$ & 54 & $89: 11$ \\
3 & $\mathbf{2 e}$ & $4-\mathrm{NCC}_{6} \mathrm{H}_{4}$ & 78 & $88: 12$ \\
4 & $\mathbf{2 g}$ & $2-$ pyridyl $^{\mathrm{g}}$ & 49 & $85: 15$ \\
5 & $\mathbf{2 h}$ & $3-\mathrm{BrC}_{6} \mathrm{H}_{4}$ & 66 & $85: 15$ \\
6 & $\mathbf{2 i}$ & $2-\mathrm{BrC}_{6} \mathrm{H}_{4}$ & 54 & $83: 17$ \\
7 & $\mathbf{2 j}$ & $\mathrm{Ph}^{2}$ & 41 & $83: 17$ \\
8 & $\mathbf{2 k}$ & $3-\mathrm{MeOC}_{6} \mathrm{H}_{4}$ & $44^{\mathrm{d}}$ & $83: 17$ \\
\hline
\end{tabular}

${ }^{\text {a }}$ Reaction conditions: chloride 4 ( 1 equiv), imine 2 (1.2 equiv) and TDAE ( 1 equiv) in anhydrous DMF, $-20^{\circ} \mathrm{C}, 1 \mathrm{~h}$, then r.t., $20 \mathrm{~h}$.

${ }^{\mathrm{b}}$ Combined yield of diastereoisomers after purification by chromatography.

${ }^{\mathrm{c}}$ Determined after separation of the two diastereoisomers by chromatography.

${ }^{\mathrm{d}}$ Imine $\mathbf{2 k}$ ( 1 equiv), chloride 4 ( 3 equiv), TDAE (3 equiv) were used.

fied by silica gel chromatography to give the two diastereoisomers of amine products $\mathbf{3}$ or $\mathbf{5}$.

(R)- $N$-[1-(4-Nitrophenyl)-2-(2-nitrophenyl)ethyl]-2-methylpropane-2-sulfinamide (3a)

Eluent: EtOAc-PE $(6: 4 \rightarrow 1: 0)$. Major diastereoisomer $(R, R)$.

Yield: $52 \mathrm{mg}(66 \%)$; yellow solid; $\mathrm{mp} 152-154{ }^{\circ} \mathrm{C}$.

${ }^{1} \mathrm{H}$ NMR $\left(200 \mathrm{MHz}, \mathrm{CDCl}_{3}\right): \delta=8.20(\mathrm{~d}, J=8.8 \mathrm{~Hz}, 2 \mathrm{H}), 7.96(\mathrm{dd}$, $J=8.0,1.4 \mathrm{~Hz}, 1 \mathrm{H}), 7.55-7.38(\mathrm{~m}, 4 \mathrm{H}), 7.29-7.24(\mathrm{~m}, 1 \mathrm{H}), 4.86-$ $4.75(\mathrm{~m}, 1 \mathrm{H}), 3.89(\mathrm{~d}, J=7.4 \mathrm{~Hz}, 1 \mathrm{H}), 3.53(\mathrm{dd}, J=13.6,8.6 \mathrm{~Hz}$, $1 \mathrm{H}), 3.34$ (dd, $J=13.6,6.2 \mathrm{~Hz}, 1 \mathrm{H}), 1.05(\mathrm{~s}, 9 \mathrm{H})$.

${ }^{13} \mathrm{C} \mathrm{NMR}\left(50 \mathrm{MHz}, \mathrm{CDCl}_{3}\right): \delta=149.6,149.2,147.6,133.2,132.3$, $128.4,127.9,125.2,124.1,60.7,56.5,40.8,22.3$ (1 carbon signal missing due to overlap).

HRMS (ESI): $m / z[\mathrm{M}+\mathrm{H}]^{+}$calcd for $\left[\mathrm{C}_{18} \mathrm{H}_{22} \mathrm{~N}_{3} \mathrm{O}_{5} \mathrm{~S}\right]^{+}: 392.1275$; found: 392.1277 .

(R)- $N$-[1-(3-Nitrophenyl)-2-(2-nitrophenyl)ethyl]-2-methylpropane-2-sulfinamide (3b)

Eluent: EtOAc-PE $(6: 4 \rightarrow 1: 0)$. Major diastereoisomer $(R, R)$.

Yield: $47 \mathrm{mg}$ (60\%); yellow solid; $\mathrm{mp} 153-154{ }^{\circ} \mathrm{C}$.

${ }^{1} \mathrm{H}$ NMR $\left(200 \mathrm{MHz}, \mathrm{CDCl}_{3}\right): \delta=8.22(\mathrm{~s}, 1 \mathrm{H}), 8.14(\mathrm{~d}, J=8.2 \mathrm{~Hz}$, $1 \mathrm{H}), 7.96(\mathrm{dd}, J=8.0,1.2 \mathrm{~Hz}, 1 \mathrm{H}), 7.71(\mathrm{~d}, J=7.6 \mathrm{~Hz}, 1 \mathrm{H}), 7.57-$ 7.29 (m, $4 \mathrm{H}), 4.86-4.75$ (m, $1 \mathrm{H}), 3.99$ (d, $J=7.4 \mathrm{~Hz}, 1 \mathrm{H}), 3.55$ (dd, $J=13.6,8.8 \mathrm{~Hz}, 1 \mathrm{H}), 3.36(\mathrm{dd}, J=13.6,6.2 \mathrm{~Hz}, 1 \mathrm{H}), 1.05$ (s, $9 \mathrm{H})$. 
${ }^{13} \mathrm{C} \mathrm{NMR}\left(50 \mathrm{MHz}, \mathrm{CDCl}_{3}\right): \delta=149.6,148.4,144.1,133.4,133.3$, $133.2,132.4,129.9,128.3,125.2,123.2,121.8,60.7,56.5,40.7$, 22.3.

HRMS (ESI): $m / z[\mathrm{M}+\mathrm{H}]^{+}$calcd for $\left[\mathrm{C}_{18} \mathrm{H}_{22} \mathrm{~N}_{3} \mathrm{O}_{5} \mathrm{~S}\right]^{+}: 392.1275$; found: 392.1274 .

(R)- $N$-[1-(2-Nitrophenyl)-2-(2-nitrophenyl)ethyl]-2-methylpropane-2-sulfinamide (3c)

Eluent: EtOAc-PE $(6: 4 \rightarrow 1: 0)$. Major diastereoisomer $(R, R)$.

Yield: $46 \mathrm{mg}$ (59\%); yellow solid; $\mathrm{mp} 69-71^{\circ} \mathrm{C}$.

${ }^{1} \mathrm{H}$ NMR $\left(200 \mathrm{MHz}, \mathrm{CDCl}_{3}\right): \delta=8.00-7.90(\mathrm{~m}, 2 \mathrm{H}), 7.74-7.59(\mathrm{~m}$, $4 \mathrm{H}), 7.50-7.42(\mathrm{~m}, 2 \mathrm{H}), 5.34-5.22(\mathrm{~m}, 1 \mathrm{H}), 4.68(\mathrm{~d}, J=9.8 \mathrm{~Hz}$, $1 \mathrm{H}), 3.55(\mathrm{dd}, J=13.6,10.0 \mathrm{~Hz}, 1 \mathrm{H}), 3.36(\mathrm{dd}, J=13.8,5.0 \mathrm{~Hz}$, $1 \mathrm{H}), 0.92(\mathrm{~s}, 9 \mathrm{H})$

${ }^{13} \mathrm{C}$ NMR (50 MHz, $\left.\mathrm{CDCl}_{3}\right): \delta=150.0,148.1,137.9,134.0,133.4$, $133.0,132.6,129.9,128.8,128.1,125.2,124.7,59.0,56.6,39.0$, 22.2.

HRMS (ESI): $m / z[\mathrm{M}+\mathrm{H}]^{+}$calcd for $\left[\mathrm{C}_{18} \mathrm{H}_{22} \mathrm{~N}_{3} \mathrm{O}_{5} \mathrm{~S}\right]^{+}: 392.1275$; found: 392.1276 .

(R)- $N$-[1-(4-Bromophenyl)-2-(2-nitrophenyl)ethyl]-2-methylpropane-2-sulfinamide (3d)

Eluent: EtOAc-PE $(6: 4 \rightarrow 1: 0)$. Major diastereoisomer $(R, R)$.

Yield: $44 \mathrm{mg}$ (52\%); yellow solid; $\mathrm{mp} 153-155^{\circ} \mathrm{C}$.

${ }^{1} \mathrm{H}$ NMR $\left(200 \mathrm{MHz}, \mathrm{CDCl}_{3}\right): \delta=7.92(\mathrm{dd}, J=8.2,1.4 \mathrm{~Hz}, 1 \mathrm{H})$, 7.49-7.37 (m, $4 \mathrm{H}), 7.24-7.17(\mathrm{~m}, 3 \mathrm{H}), 4.70-4.60(\mathrm{~m}, 1 \mathrm{H}), 3.68$ $(\mathrm{d}, J=6.0 \mathrm{~Hz}, 1 \mathrm{H}), 3.55(\mathrm{dd}, J=13.6,8.2 \mathrm{~Hz}, 1 \mathrm{H}), 3.29(\mathrm{dd}, J=$ 13.6, $6.6 \mathrm{~Hz}, 1 \mathrm{H}), 1.07(\mathrm{~s}, 9 \mathrm{H})$.

${ }^{13} \mathrm{C} \mathrm{NMR}\left(50 \mathrm{MHz}, \mathrm{CDCl}_{3}\right): \delta=140.7,133.2,132.9,132.7,132.0$, $131.8,128.7,128.0,125.0,122.1,60.4,56.2,40.6,22.4$.

HRMS (ESI): $m / z[\mathrm{M}+\mathrm{H}]^{+}$calcd for $\left[\mathrm{C}_{18} \mathrm{H}_{22} \mathrm{BrN}_{2} \mathrm{O}_{3} \mathrm{~S}\right]^{+}: 425.0529$; found: 425.0529 .

\section{(R)-N-[1-(4-Cyanophenyl)-2-(2-nitrophenyl)ethyl]-2-methyl-} propane-2-sulfinamide (3e)

Eluent: EtOAc-PE $(6: 4 \rightarrow 1: 0)$. Major diastereoisomer $(R, R)$.

Yield: $46 \mathrm{mg}$ (62\%); yellow solid; $\mathrm{mp} 175-177^{\circ} \mathrm{C}$.

${ }^{1} \mathrm{H}$ NMR $\left(300 \mathrm{MHz}, \mathrm{CDCl}_{3}\right): \delta=7.94(\mathrm{dd}, J=8.1,1.2 \mathrm{~Hz}, 1 \mathrm{H})$, $7.62(\mathrm{~d}, J=8.4 \mathrm{~Hz}, 2 \mathrm{H}), 7.54-7.38(\mathrm{~m}, 4 \mathrm{H}), 7.25(\mathrm{~d}, J=7.5 \mathrm{~Hz}$, $1 \mathrm{H}), 4.77-4.69(\mathrm{~m}, 1 \mathrm{H}), 3.93(\mathrm{~d}, J=7.5 \mathrm{~Hz}, 1 \mathrm{H}), 3.49(\mathrm{dd}, J=$ 13.5, 8.7 Hz, 1 H), 3.32 (dd, $J=13.5,6.3 \mathrm{~Hz}, 1 \mathrm{H}), 1.04(\mathrm{~s}, 9 \mathrm{H})$.

${ }^{13} \mathrm{C} \mathrm{NMR}\left(75 \mathrm{MHz}, \mathrm{CDCl}_{3}\right): \delta=149.6,147.2,133.2,133.1,132.6$, 132.4, 128.3, 127.8, 125.1, 118.5, 112.0, 60.9, 56.4, 40.7, 22.3.

HRMS (ESI): $m / z[\mathrm{M}+\mathrm{H}]^{+}$calcd for $\left[\mathrm{C}_{19} \mathrm{H}_{22} \mathrm{~N}_{3} \mathrm{O}_{3} \mathrm{~S}\right]^{+}: 372.1376$; found: 372.1378 .

(R)- $N$-[1-(3-cyanophenyl)-2-(2-nitrophenyl)ethyl]-2-methylpropane-2-sulfinamide (3f)

Eluent: EtOAc-PE $(6: 4 \rightarrow 1: 0)$. Major diastereoisomer $(R, R)$.

Yield: $48 \mathrm{mg}$ (65\%); yellow solid; $\mathrm{mp} 97-99{ }^{\circ} \mathrm{C}$.

${ }^{1} \mathrm{H}$ NMR $\left(400 \mathrm{MHz}, \mathrm{CDCl}_{3}\right): \delta=7.95(\mathrm{~d}, J=8.2 \mathrm{~Hz}, 1 \mathrm{H}), 7.61-$ $7.58(\mathrm{~m}, 3 \mathrm{H}), 7.52-7.41(\mathrm{~m}, 4 \mathrm{H}), 4.75-4.70(\mathrm{~m}, 1 \mathrm{H}), 3.84(\mathrm{~d}, J=$ $6.8 \mathrm{~Hz}, 1 \mathrm{H}), 3.53(\mathrm{dd}, J=13.6,8.4 \mathrm{~Hz}, 1 \mathrm{H}), 3.32(\mathrm{dd}, J=13.6$, $6.0 \mathrm{~Hz}, 1 \mathrm{H}), 1.06(\mathrm{~s}, 9 \mathrm{H})$.

${ }^{13} \mathrm{C} \mathrm{NMR}\left(100 \mathrm{MHz}, \mathrm{CDCl}_{3}\right): \delta=149.7,143.4,133.2,133.1,132.3$, $131.8,131.6,130.5,129.7,128.3,125.2,118.5,112.9,60.5,56.4$, 40.7, 22.3.

HRMS (ESI): $m / z[\mathrm{M}+\mathrm{H}]^{+}$calcd for $\left[\mathrm{C}_{19} \mathrm{H}_{22} \mathrm{~N}_{3} \mathrm{O}_{3} \mathrm{~S}\right]^{+}: 372.1376$; found: 372.1377 .

(R)- $N$-[1-(Pyridin-2-yl)-2-(2-nitrophenyl)ethyl]-2-methylpropane-2-sulfinamide (3g)

Eluent: EtOAc-PE $(6: 4 \rightarrow 1: 0)$. Major diastereoisomer $(R, R)$.

Yield: $41 \mathrm{mg}$ (59\%); yellow oil.
${ }^{1} \mathrm{H}$ NMR $\left(200 \mathrm{MHz}, \mathrm{CDCl}_{3}\right): \delta=8.58(\mathrm{~d}, J=4.6 \mathrm{~Hz}, 1 \mathrm{H}), 7.98(\mathrm{~d}$, $J=8.0 \mathrm{~Hz}, 1 \mathrm{H}), 7.73-7.66(\mathrm{~m}, 1 \mathrm{H}), 7.48-7.28(\mathrm{~m}, 5 \mathrm{H}), 5.18(\mathrm{~d}$, $J=8.8 \mathrm{~Hz}, 1 \mathrm{H}), 4.86-4.75(\mathrm{~m}, 1 \mathrm{H}), 3.46(\mathrm{dd}, J=13.4,5.4 \mathrm{~Hz}$, $1 \mathrm{H}), 3.30(\mathrm{dd}, J=13.4,9.2 \mathrm{~Hz}, 1 \mathrm{H}), 1.09(\mathrm{~s}, 9 \mathrm{H})$.

${ }^{13} \mathrm{C}$ NMR $\left(50 \mathrm{MHz}, \mathrm{CDCl}_{3}\right): \delta=160.4,149.6,149.1,137.4,134.2$, 133.4, 132.8, 128.0, 125.0, 123.0, 122.3, 61.7, 56.2, 41.9, 22.7.

HRMS (ESI): $m / z[\mathrm{M}+\mathrm{H}]^{+}$calcd for $\left[\mathrm{C}_{17} \mathrm{H}_{22} \mathrm{~N}_{3} \mathrm{O}_{3} \mathrm{~S}\right]^{+}: 348.1376$; found: 348.1374 .

(R)- $N$-\{2-[4-(1,2-Dimethyl-5-nitro-1H-imidazol-4-yl)phenyl]-1(4-nitrophenyl)ethyl\}-2-methyl Propane-2-sulfinamide (5a) Eluent: EtOAc-PE $(6: 4 \rightarrow 1: 0)$. Major diastereoisomer $(R, R)$.

Yield: $55 \mathrm{mg}$ (57\%); yellow solid; $\mathrm{mp} 111-113{ }^{\circ} \mathrm{C}$.

${ }^{1} \mathrm{H}$ NMR $\left(200 \mathrm{MHz}, \mathrm{CDCl}_{3}\right): \delta=8.15(\mathrm{~d}, J=8.8 \mathrm{~Hz}, 2 \mathrm{H}), 7.62(\mathrm{~d}$, $J=8.0 \mathrm{~Hz}, 2 \mathrm{H}), 7.42(\mathrm{~d}, J=8.0 \mathrm{~Hz}, 2 \mathrm{H}), 7.06(\mathrm{~d}, J=8.0 \mathrm{~Hz}, 2 \mathrm{H})$, $4.78-4.69(\mathrm{~m}, 1 \mathrm{H}), 3.90(\mathrm{~s}, 3 \mathrm{H}), 3.72(\mathrm{~d}, J=5.2 \mathrm{~Hz}, 1 \mathrm{H}), 3.34(\mathrm{dd}$, $J=13.6,6.8 \mathrm{~Hz}, 1 \mathrm{H}), 3.07$ (dd, $J=13.6,7.6 \mathrm{~Hz}, 1 \mathrm{H}), 2.51(\mathrm{~s}, 3 \mathrm{H})$, $1.16(\mathrm{~s}, 9 \mathrm{H})$.

${ }^{13} \mathrm{C}$ NMR (75 MHz, $\mathrm{CDCl}_{3}$ ): $\delta=149.0,148.4,147.5,143.1,137.7$, $134.7,130.6,129.7,129.2,128.3,123.8,60.5,56.5,43.4,34.2$, $22.5,14.2$.

HRMS (ESI): $m / z[\mathrm{M}+\mathrm{H}]^{+}$calcd for $\left[\mathrm{C}_{23} \mathrm{H}_{28} \mathrm{~N}_{5} \mathrm{O}_{5} \mathrm{~S}\right]^{+}: 486.1806$; found: 486.1805 .

(R)- $N$-\{2-[4-(1,2-Dimethyl-5-nitro- $1 H$-imidazol-4-yl)phenyl]-1(4-bromophenyl)ethyl\}-2-methyl propane-2-sulfinamide (5d)

Eluent: EtOAc-PE (6:4) $\rightarrow$ EtOAc-MeOH (40:1). Major diastereoisomer $(R, R)$.

Yield: $56 \mathrm{mg}$ (54\%); yellow solid; $\mathrm{mp} 87-89^{\circ} \mathrm{C}$.

${ }^{1} \mathrm{H}$ NMR $\left(200 \mathrm{MHz}, \mathrm{CDCl}_{3}\right): \delta=7.60(\mathrm{~d}, J=8.2 \mathrm{~Hz}, 2 \mathrm{H}), 7.40(\mathrm{~d}$, $J=8.2 \mathrm{~Hz}, 2 \mathrm{H}), 7.12(\mathrm{~d}, J=8.4 \mathrm{~Hz}, 2 \mathrm{H}), 7.06(\mathrm{~d}, J=8.4 \mathrm{~Hz}, 2 \mathrm{H})$, $4.62-4.52(\mathrm{~m}, 1 \mathrm{H}), 3.88(\mathrm{~s}, 3 \mathrm{H}), 3.71(\mathrm{~d}, J=4.6 \mathrm{~Hz}, 1 \mathrm{H}), 3.29$ (dd, $J=13.4,6.6 \mathrm{~Hz}, 1 \mathrm{H}), 3.00(\mathrm{dd}, J=13.4,7.6 \mathrm{~Hz}, 1 \mathrm{H}), 2.50(\mathrm{~s}, 3 \mathrm{H})$, 1.14 (s, $9 \mathrm{H})$.

${ }^{13} \mathrm{C}$ NMR $\left(75 \mathrm{MHz}, \mathrm{CDCl}_{3}\right): \delta=148.3,143.3,140.6,138.5,131.7$, 130.2, 129.5, 129.3, 129.0, 121.8, 60.3, 56.2, 43.3, 34.2, 22.5, 14.2; 1 carbon signal missing due to overlap.

HRMS (ESI): $m / z[\mathrm{M}+\mathrm{H}]^{+}$calcd for $\left[\mathrm{C}_{23} \mathrm{H}_{28} \mathrm{BrN}_{4} \mathrm{O}_{3} \mathrm{~S}\right]^{+}: 519.1060$; found: 519.1060 .

(R)- $N$-\{2-[4-(1,2-Dimethyl-5-nitro- $1 H$-imidazol-4-yl)phenyl]-1(4-cyanophenyl)ethyl\}-2-methyl propane-2-sulfinamide (5e)

Eluent: EtOAc-PE (6:4) $\rightarrow$ EtOAc-MeOH (20:1). Major diastereoisomer $(R, R)$.

Yield: $73 \mathrm{mg}$ (78\%); yellow solid; $\mathrm{mp} 112-114^{\circ} \mathrm{C}$.

${ }^{1} \mathrm{H}$ NMR $\left(200 \mathrm{MHz}, \mathrm{CDCl}_{3}\right): \delta=7.61-7.54(\mathrm{~m}, 4 \mathrm{H}), 7.34(\mathrm{~d}, J=$ $8.2 \mathrm{~Hz}, 2 \mathrm{H}), 7.04$ (d, $J=8.0 \mathrm{~Hz}, 2 \mathrm{H}), 4.70-4.60(\mathrm{~m}, 1 \mathrm{H}), 3.87$ (s, $3 \mathrm{H}), 3.77(\mathrm{~d}, J=5.4 \mathrm{~Hz}, 1 \mathrm{H}), 3.29(\mathrm{dd}, J=13.4,7.0 \mathrm{~Hz}, 1 \mathrm{H}), 3.02$ (dd, $J=13.4,7.2 \mathrm{~Hz}, 1 \mathrm{H}), 2.49$ (s, $3 \mathrm{H}), 1.12$ (s, $9 \mathrm{H})$.

${ }^{13} \mathrm{C} \mathrm{NMR}\left(75 \mathrm{MHz}, \mathrm{CDCl}_{3}\right): \delta=148.3,146.9,143.1,137.8,132.4$, 130.6, 129.6, 129.2, 128.1, 118.6, 111.8, 60.6, 56.4, 43.3, 34.2, $22.5,14.2 ; 1$ carbon signal missing due to overlap.

HRMS (ESI): $m / z[\mathrm{M}+\mathrm{H}]^{+}$calcd for $\left[\mathrm{C}_{24} \mathrm{H}_{28} \mathrm{~N}_{5} \mathrm{O}_{3} \mathrm{~S}\right]^{+}: 466.1907$; found: 466.1906 .

(R)- $N$-\{2-[4-(1,2-dimethyl-5-nitro-1H-imidazol-4-yl)phenyl]-1(pyridin-2-yl)ethyl $\}$-2-methyl propane-2-sulfinamide (5g) Eluent: EtOAc-PE (6:4) $\rightarrow$ EtOAc-MeOH (9:1). Major diastereoisomer $(R, R)$.

Yield: $43 \mathrm{mg}$ (49\%); yellow oil.

${ }^{1} \mathrm{H}$ NMR $\left(300 \mathrm{MHz}, \mathrm{CDCl}_{3}\right): \delta=8.56(\mathrm{~d}, J=4.5 \mathrm{~Hz}, 1 \mathrm{H}), 7.62$ $7.55(\mathrm{~m}, 3 \mathrm{H}), 7.20-7.10(\mathrm{~m}, 4 \mathrm{H}), 4.88(\mathrm{~d}, J=7.8 \mathrm{~Hz}, 1 \mathrm{H}), 4.70$ $4.63(\mathrm{~m}, 1 \mathrm{H}), 3.87(\mathrm{~s}, 3 \mathrm{H}), 3.16(\mathrm{~d}, J=7.2, \mathrm{~Hz}, 2 \mathrm{H}), 2.49(\mathrm{~s}, 3 \mathrm{H})$, $1.14(\mathrm{~s}, 9 \mathrm{H})$. 
${ }^{13} \mathrm{C}$ NMR $\left(75 \mathrm{MHz}, \mathrm{CDCl}_{3}\right): \delta=160.4,149.0,148.4,143.6,139.2$, $137.2,134.9,130.2,129.5,129.4,122.8,122.6,62.4,56.3,44.3$, $34.3,22.8,14.3$

HRMS (ESI): $m / z[\mathrm{M}+\mathrm{H}]^{+}$calcd for $\left[\mathrm{C}_{22} \mathrm{H}_{28} \mathrm{~N}_{5} \mathrm{O}_{3} \mathrm{~S}\right]^{+}: 442.1907$; found: 442.1908 .

(R)- $N$-\{2-[4-(1,2-dimethyl-5-nitro-1H-imidazol-4-yl)phenyl]-1(3-bromophenyl)ethyl\}-2-methyl propane-2-sulfinamide (5h) Eluent: EtOAc-PE (6:4) $\rightarrow$ EtOAc-MeOH (40:1). Major diastereoisomer $(R, R)$.

Yield: $69 \mathrm{mg}$ (66\%); yellow solid; $\mathrm{mp} 107-109^{\circ} \mathrm{C}$.

${ }^{1} \mathrm{H} \mathrm{NMR}\left(200 \mathrm{MHz}, \mathrm{CDCl}_{3}\right): \delta=7.60(\mathrm{~d}, J=8.2 \mathrm{~Hz}, 2 \mathrm{H}), 7.47(\mathrm{~s}$, $1 \mathrm{H}), 7.40-7.35(\mathrm{~m}, 1 \mathrm{H}), 7.16-7.09(\mathrm{~m}, 4 \mathrm{H}), 4.58-4.48(\mathrm{~m}, 1 \mathrm{H})$, $4.14(\mathrm{~d}, J=6.4 \mathrm{~Hz}, 1 \mathrm{H}), 3.87(\mathrm{~s}, 3 \mathrm{H}), 3.25(\mathrm{dd}, J=13.6,7.4 \mathrm{~Hz}$, $1 \mathrm{H}), 3.03(\mathrm{dd}, J=13.6,7.2 \mathrm{~Hz}, 1 \mathrm{H}), 2.49(\mathrm{~s}, 3 \mathrm{H}), 1.10(\mathrm{~s}, 9 \mathrm{H})$.

${ }^{13} \mathrm{C}$ NMR (75 MHz, $\mathrm{CDCl}_{3}$ ): $\delta=148.3,144.0,143.3,138.4,134.8$, $131.1,130.3,130.2,130.1,129.5,129.3,126.3,122.7,60.4,56.2$, $43.4,34.2,22.5,14.2$.

HRMS (ESI): $m / z[\mathrm{M}+\mathrm{H}]^{+}$calcd for $\left[\mathrm{C}_{23} \mathrm{H}_{28} \mathrm{BrN}_{4} \mathrm{O}_{3} \mathrm{~S}\right]^{+}: 519.1060$; found: 519.1063 .

(R)- $N$-\{2-[4-(1,2-Dimethyl-5-nitro-1H-imidazol-4-yl)phenyl]-1(2-bromophenyl)ethyl\}-2-methyl propane-2-sulfinamide (5i) Eluent: EtOAc-PE (6:4) $\rightarrow$ EtOAc-MeOH (40:1). Major diastereoisomer $(R, R)$.

Yield: $56 \mathrm{mg}$ (54\%); yellow solid; $\mathrm{mp} 113-115^{\circ} \mathrm{C}$.

${ }^{1} \mathrm{H}$ NMR $\left(200 \mathrm{MHz}, \mathrm{CDCl}_{3}\right): \delta=7.65(\mathrm{~d}, J=8.2 \mathrm{~Hz}, 2 \mathrm{H}), 7.54(\mathrm{~d}$, $J=7.8 \mathrm{~Hz}, 1 \mathrm{H}), 7.42-7.12(\mathrm{~m}, 5 \mathrm{H}), 5.07-4.97(\mathrm{~m}, 1 \mathrm{H}), 3.96(\mathrm{~d}$, $J=6.6 \mathrm{~Hz}, 1 \mathrm{H}), 3.90(\mathrm{~s}, 3 \mathrm{H}), 3.24(\mathrm{dd}, J=13.6,5.4 \mathrm{~Hz}, 1 \mathrm{H}), 3.04$ $(\mathrm{dd}, J=13.6,8.2 \mathrm{~Hz}, 1 \mathrm{H}), 2.51(\mathrm{~s}, 3 \mathrm{H}), 1.05(\mathrm{~s}, 9 \mathrm{H})$.

${ }^{13} \mathrm{C}$ NMR $\left(50 \mathrm{MHz}, \mathrm{CDCl}_{3}\right): \delta=148.3,143.4,140.7,138.8,133.2$, $130.3,129.6,129.4,129.2,128.8,127.8,123.2,60.5,56.6,42.6$, $34.2,22.4,14.2 ; 1$ carbon signal missing due to overlap.

HRMS (ESI): $m / z[\mathrm{M}+\mathrm{H}]^{+}$calcd for $\left[\mathrm{C}_{23} \mathrm{H}_{28} \mathrm{~N}_{4} \mathrm{O}_{3} \mathrm{SBr}\right]^{+}: 519.1060$; found: 519.1063 .

(R)-N-(2-(4-(1,2-dimethyl-5-nitro-1H-imidazol-4-yl)phenyl)-1(phenyl)ethyl)-2-methyl propane-2-sulfinamide (5j)

Eluent: EtOAc-PE (6:4) $\rightarrow$ EtOAc-MeOH (9:1). Major diastereoisomer $(R, R)$.

Yield: $36 \mathrm{mg}(41 \%)$; yellow solid; $\mathrm{mp} 82-84{ }^{\circ} \mathrm{C}$.

${ }^{1} \mathrm{H}$ NMR $\left(200 \mathrm{MHz}, \mathrm{CDCl}_{3}\right): \delta=7.60(\mathrm{~d}, J=8.2 \mathrm{~Hz}, 2 \mathrm{H}), 7.28$ $7.24(\mathrm{~m}, 5 \mathrm{H}), 7.07(\mathrm{~d}, J=8.2 \mathrm{~Hz}, 2 \mathrm{H}), 4.67-4.58(\mathrm{~m}, 1 \mathrm{H}), 3.89(\mathrm{~s}$, $3 \mathrm{H}), 3.59(\mathrm{~d}, J=3.8 \mathrm{~Hz}, 1 \mathrm{H}), 3.34(\mathrm{dd}, J=13.4,6.6 \mathrm{~Hz}, 1 \mathrm{H}), 3.06$ $(\mathrm{dd}, J=13.4,7.6 \mathrm{~Hz}, 1 \mathrm{H}), 2.51(\mathrm{~s}, 3 \mathrm{H}), 1.16(\mathrm{~s}, 9 \mathrm{H})$.

${ }^{13} \mathrm{C}$ NMR (75 MHz, $\mathrm{CDCl}_{3}$ ): $\delta=148.3,143.5,141.5,139.0,130.0$, $129.4,129.3,128.6,127.9,127.8,127.3,60.7,56.1,43.5,34.1$, 22.6, 14.2.

HRMS (ESI): $m / z[\mathrm{M}+\mathrm{H}]^{+}$calcd for $\left[\mathrm{C}_{23} \mathrm{H}_{29} \mathrm{~N}_{4} \mathrm{O}_{3} \mathrm{~S}\right]^{+}: 441.1955$; found: 441.1957.

(R)- $N$-\{2-[4-(1,2-Dimethyl-5-nitro-1H-imidazol-4-yl)phenyl]-1(3-methoxyphenyl)ethyl\}-2-methyl Propane-2-sulfinamide (5k) Eluent: EtOAc-PE (6:4) $\rightarrow$ EtOAc-MeOH (40:1). Major diastereoisomer $(R, R)$.

Yield: $39 \mathrm{mg}$ (44\%); yellow solid; mp $92-94{ }^{\circ} \mathrm{C}$

${ }^{1} \mathrm{H} \mathrm{NMR}\left(400 \mathrm{MHz}, \mathrm{CDCl}_{3}\right): \delta=7.60(\mathrm{~d}, J=8.4 \mathrm{~Hz}, 2 \mathrm{H}), 7.19(\mathrm{dd}$, $J=8.0,7.6 \mathrm{~Hz}, 1 \mathrm{H}), 7.08(\mathrm{~d}, J=8.0 \mathrm{~Hz}, 2 \mathrm{H}), 6.85-6.77(\mathrm{~m}, 3 \mathrm{H})$, $4.61-4.57(\mathrm{~m}, 1 \mathrm{H}), 3.88(\mathrm{~s}, 3 \mathrm{H}), 3.74(\mathrm{~s}, 3 \mathrm{H}), 3.58(\mathrm{~d}, J=4.0 \mathrm{~Hz}$, $1 \mathrm{H}), 3.30(\mathrm{dd}, J=13.4,6.6 \mathrm{~Hz}, 1 \mathrm{H}), 3.05(\mathrm{dd}, J=13.4,7.8 \mathrm{~Hz}$, $1 \mathrm{H}), 2.49$ (s, $3 \mathrm{H}), 1.15$ (s, $9 \mathrm{H})$.
${ }^{13} \mathrm{C}$ NMR $\left(100 \mathrm{MHz}, \mathrm{CDCl}_{3}\right): \delta=159.7,148.3,143.5,143.2,139.0$, $130.1,129.6,129.39,129.37,119.5,113.4,112.9,60.7,56.1,55.2$, $43.4,34.2,22.6,14.2 ; 1$ carbon signal missing due to overlap.

HRMS (ESI): $m / z[\mathrm{M}+\mathrm{H}]^{+}$calcd for $\left[\mathrm{C}_{24} \mathrm{H}_{31} \mathrm{~N}_{4} \mathrm{O}_{4} \mathrm{~S}\right]^{+}: 471.2061$; found: 471.2060 .

\section{Acknowledgment}

This work was supported by the CNRS and the Aix-Marseille University. The authors thank the Spectropole team for HRMS analysis. We express our thanks to V. Remusat for recording ${ }^{1} \mathrm{H}$ and ${ }^{13} \mathrm{C}$ NMR spectra.

\section{References}

(1) Hili, R.; Yudin, A. K. Nat. Chem. Biol. 2006, 2, 284.

(2) Robak, M. T.; Herbage, M. A.; Ellman, J. A. Chem. Rev. 2010, 110,3600

(3) Buesking, A. W.; Baguley, T. D.; Ellman, J. A. Org. Lett. 2011, 13, 964

(4) Sapountzis, I.; Dube, H.; Lewis, R.; Gommermann, N.; Knochel, P. J. Org. Chem. 2005, 70, 2445.

(5) (a) Takechi, N.; Aït-Mohand, S.; Médebielle, M.; Dolbier, W. R. Jr. Tetrahedron Lett. 2002, 43, 4317. (b) Pooput, C.; Médebielle, M.; Dolbier, W. R. Jr. Org. Lett. 2004, 6, 301. (c) Pooput, C.; Médebielle, M.; Dolbier, W. R. Jr. J. Org. Chem. 2006, 71, 3564.

(6) (a) Montana, M.; Terme, T.; Vanelle, P. Tetrahedron Lett. 2005, 46, 8373. (b) Amiri-Attou, O.; Terme, T.; Vanelle, P. Molecules 2005, 10, 545. (c) Montana, M.; Terme, T.; Vanelle, P. Tetrahedron Lett. 2006, 47, 6573. (d) Juspin, T.; Terme, T.; Vanelle, P. Synlett 2009, 1485. (e) NadjiBoukrouche, A. R.; Khoumeri, O.; Terme, T.; Liacha, M.; Vanelle, P. ARKIVOC 2010, (x), 358. (f) Montana, M.; Terme, T.; Vanelle, P. Lett. Org. Chem. 2010, 7, 453. (g) Juspin, T.; Giuglio-Tonolo, G.; Terme, T.; Vanelle, P. Synthesis 2010, 844.

(7) Spitz, C.; Khoumeri, O.; Terme, T.; Vanelle, P. Synlett 2013, $24,1725$.

(8) (a) Crozet, M. P.; Archaimbault, G.; Vanelle, P.; Nouguier, R. Tetrahedron Lett. 1985, 26, 5133. (b) Curti, C.; Laget, M.; Ortiz Carle, A.; Gellis, A.; Vanelle, P. Eur. J. Med. Chem. 2007, 42, 880. (c) Cohen, A.; Crozet, M. D.; Rathelot, P.; Vanelle, P. Green Chem. 2009, 11, 1736. (d) Verhaeghe, P.; Azas, N.; Hutter, S.; Castera-Ducros, C.; Laget, M.; Dumètre, A.; Gasquet, M.; Reboul, J.-P.; Rault, S.; Rathelot, P.; Vanelle, P. Bioorg. Med. Chem. 2009, 17, 4313.

(9) (a) Jorgensen, M. A.; Manos, J.; Mendz, G. L.; Hazell, S. L. J. Antimicrob. Chemother. 1998, 41, 67. (b) Upcroft, J. A.; Campbell, R. W.; Benakli, K.; Upcroft, P.; Vanelle, P. Antimicrob. Agents Chemother. 1999, 43, 73. (c) Citron, D. M.; Tyrrell, K. L.; Warren, Y. A.; Fernandez, H.; Merriam, C. V.; Goldstein, E. J. C. Anaerobe 2005, 11, 315.

(d) Leitsch, D.; Kolarich, D.; Wilson, I. B. H.; Altmann, F.; Duchêne, M. PLoS Biol. 2007, 5, e211. (e) Crozet, M. D.; Botta, C.; Gasquet, M.; Curti, C.; Rémusat, V.; Hutter, S.; Chapelle, O.; Azas, N.; De Méo, M.; Vanelle, P. Eur. J. Med. Chem. 2009, 44, 653. (f) Kim, P.; Kang, S.; Boshoff, H. I.; Jiricek, J.; Collins, M.; Singh, R.; Manjunatha, U. H.; Niyomrattanakit, P.; Zhang, L.; Goodwin, M.; Dick, T.; Keller, T. H.; Dowd, C. S.; Barry, C. E. III J. Med. Chem. 2009, 52, 1329. (g) Dunn, L. A.; Burgess, A. G.; Krauer, K. G.; Eckmann, L.; Vanelle, P.; Crozet, M. D.; Gillin, F. D.; Upcroft, P.; Upcroft, J. A. Int. J. Antimicrob. Agents 2010, $36,37$. 